\title{
Charmonium dissociation temperatures in lattice QCD with a finite volume technique
}

\author{
T. Umedat, H. Ohno, K. Kanaya for the WHOT-QCD \\ Collaboration \\ Graduate School of Pure and Applied Sciences, University of Tsukuba, \\ Tsukuba, Ibaraki 305-8571, Japan \\ E-mail: tumeda@het.ph.tsukuba.ac.jp
}

\begin{abstract}
Dissociation temperatures of $J / \psi, \psi^{\prime}$, and $\chi_{c}$ states play key roles in the sequential $J / \psi$ suppression scenario for high energy heavy ion collisions. We report on a study of charmonium dissociation temperatures in quenched lattice QCD. On anisotropic lattices, we first subtract the effects of the constant mode in finite temperature meson correlators, which have lead to unphysical results in previous studies. We then extract ground and first exited state masses by diagonalizing correlation functions among different source and sink operators. To distinguish bound states from scattering states, we first compare the charmonium mass spectra under different spatial boundary conditions, and examine the shape and the volume-dependence of their Bethe-Salpeter wave functions. From these studies, we found so far no sign of scattering states up to about $2.3 T_{c}$.
\end{abstract}

\section{Introduction}

Heavy quarkona play important roles in the study of quark gluon plasma (QGP) in high energy heavy ion collisions. Lattice studies of charmonium spectral functions at finite temperatures have suggested that hadronic excitations corresponding to $J / \psi$ may survive in the deconfinement phase till relatively high temperatures [1, 2, 3, 4, 5, 6]. In order to understand discrepancy with the experimental observation of $J / \psi$ suppression, a sequential $J / \psi$ suppression scenario has been proposed [7, 8], in which a part of $J / \psi$ particles in heavy ion collisions are produced through $\psi^{\prime}$ and $\chi_{c}$ 's and thus dissociation of $\psi^{\prime}$ and $\chi_{c}$ 's states lead to additional contributions to the $J / \psi$ suppression.

To examine the scenario, several groups started to study spectral functions for $\chi_{c}$ states on the lattice adopting a Bayesian method [3, 5, 6]. They found that the low energy part of the spectral functions for $\chi_{c 0}$ and $\chi_{c 1}$ states drastically increase just above $T_{c}$, suggesting dissociation of these states immediately above $T_{c}$. On the other hand, one of the present authors has recently pointed out that such change is caused by a constant mode expected in high temperature meson correlation functions on the lattice 9 . To obtain physically meaningful results from meson correlation functions in the deconfining phase, we have to separate the effects of the constant mode.

In this paper, we carry out a study of charmonium states above $T_{c}$ in quenched QCD on anisotropic lattices, subtracting the contribution of the constant mode.

$\ddagger$ speaker 


\section{Our approach}

We combine all the known rigid techniques to investigate meson states at high temperature. In order to extract a few lowest energy states in Euclidean correlators we adopt the variational method [10], in which the eigenvalues $\lambda_{\alpha}\left(t, t_{0}\right)$ of the correlation matrix provide the energies of the states.

$$
X^{-1} C\left(t_{0}\right)^{-1} C(t) X=\operatorname{diag}\left\{\lambda_{\alpha}\left(t, t_{0}\right)\right\}, \quad(\alpha=1, \cdots, N) .
$$

The $N \times N$ correlation matrix is defined by

$$
\begin{aligned}
& C_{i j}(t)=\sum_{\vec{x}}\left\langle O_{i}(\vec{x}, t) O_{j}^{\dagger}(\overrightarrow{0}, 0)\right\rangle, \\
& O_{i}(\vec{x}, t)=\sum_{y, z} \phi_{i}(\vec{y}) \phi_{i}(\vec{z}) \bar{q}(\vec{x}+\vec{y}, t) \Gamma q(\vec{x}+\vec{z}, t)
\end{aligned}
$$

where $\Gamma=\gamma_{1}$ and $\gamma_{1} \gamma_{5}$ for vector $(\mathrm{V})$ and axial-vector $(\mathrm{Av})$ channels, respectively. In this study we use operators with Gaussian smearing function $\phi_{i}(\vec{x})=\exp \left(-A|\vec{x}|^{2}\right)$ with $N$ different values of the width parameter $A$. The analysis enables us to study excited states such as $\psi(2 S)$, which is the radial excitation state of charmonium with $J^{P C}=1^{--}$channel.

As mentioned in the Introduction, to study meson-like states in the high temperature phase, we have to subtract the effects of the constant mode from the meson correlators, because it sometimes makes it difficult to extract low energy states, in particular for P-waves. We adopt the midpoint subtraction method [9] to remove the constant mode.

To distinguish bound states of $c-\bar{c}$ quarks from their scattering states in lattice QCD, we study the spatial boundary condition dependence of the energy spectrum at finite volume [11: The energy of scattering states depends on its relative momentum which is quantized according to the volume size and boundary conditions. On the other hand the spectrum of the bound states does not change against such exchange of boundary conditions. Therefore, we can judge the existence of scattering states from a shift in the energy spectrum. In this study, we prepare three types of spatial boundary condition, $\mathrm{PBC}, \mathrm{APBC}$ and $\mathrm{MBC}$, where (A)PBC is the (anti-)periodic boundary condition in all spatial directions, and $\mathrm{MBC}$ is anti-periodic in $\mathrm{x}$-direction and periodic in the others directions.

We also study the Bethe-Salpeter wave function from the spatial correlation function between $c-\bar{c}$ quarks [1. We expect that wave functions for binding state will be compact, while those for scattering state will be extended and will change its shape depending on the lattice volume.

\section{Numerical Results}

\subsection{Lattice setup}

The gauge configurations have been generated by an standard plaquette gauge action with a lattice gauge coupling constant, $\beta=6.10$ and a bare anisotropy parameter $\gamma_{G}=3.2108$. The definition of the action and parameters are the same as that adopted in Ref. [12. The lattice spacings are $1 / a_{s}=2.030(13) \mathrm{GeV}$ and $1 / a_{t}=8.12(5)$ $\mathrm{GeV}$. Our simulations are performed on $N_{s}^{3} \times N_{t}$ lattices with $N_{t}=32-12$ and $N_{s}=16-32$ to study temperature and volume dependence respectively. Temperatures are given by $T / T_{c}=N_{t}^{c r i t} / N_{t}$ where $N_{t}^{c r i t} \sim 28$ in our setup. For the quark fields, we 

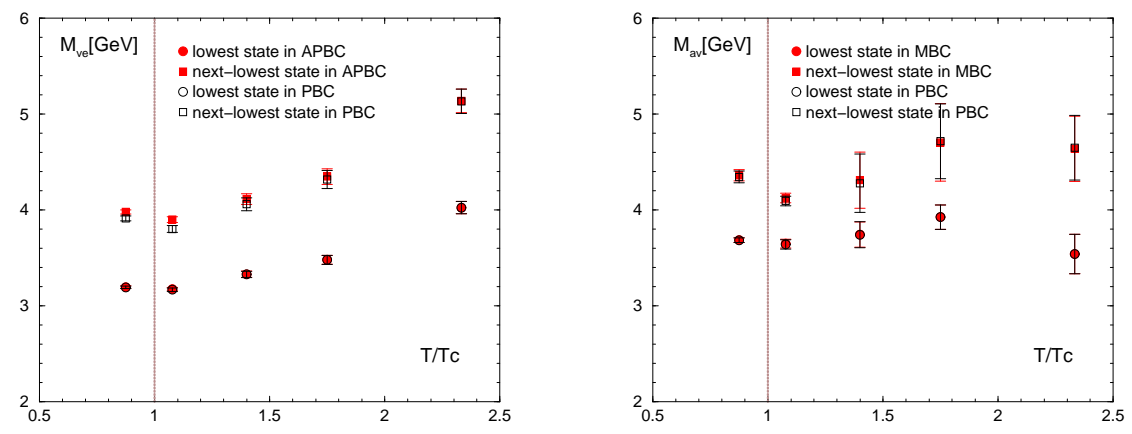

Figure 1. Temperature dependences of the lowest and next-lowest state energies in different boundary conditions. Left and right panels show charmonium with vector and axial-vector channels respectively.

adopt an $O(a)$-improved Wilson quark action with tadpole improved tree level clover coefficients. Although the definition of the quark action is the same as in Ref. [12, we adopt a different choice of the Wilson parameter $r=1$ to suppress lattice artifact in higher excited states.

\subsection{Boundary condition dependences}

Figure 1 shows the temperature dependence of charmonium energies in vector and axial-vector channels. These energies are calculated by the variational method with $4 \times 4$ correlation matrix. At $T=0$ the lowest and next-lowest states in the vector channel are $J / \psi$ and $\psi(2 S)$ states respectively. The lowest state in the axial-vector channel is the $\chi_{c 1}$ state at $T=0$. Results adopting different boundary conditions are shown by different colors.

From a study of meson-like correlators in the free quark case as a model of scattering states, we find that the largest shift of the ground state energy appears between PBC and APBC for the S-wave, while for P-wave states between $\mathrm{PBC}$ and $\mathrm{MBC}$. Therefore, we compare $\mathrm{PBC}$ and $\mathrm{APBC}(\mathrm{MBC})$ for $\mathrm{S}-(\mathrm{P}-)$ waves. As a reference, energy shifts in the lowest states are about $200 \mathrm{MeV}$ for the case of free quarks in a $(2 \mathrm{fm})^{3}$ box.

Although statistical errors in axial-vector channels are not quite small yet, Fig. 1 shows that typical mass shifts are much smaller than those expected in the free quark case up to about $2.3 T_{c}$.

\subsection{Wave functions}

Figure 2 shows the volume dependence of charmonium wave functions in vector and axial-vector channels at $T=2.3 T_{c}$. The Bethe-Salpeter wave function is defined by

$$
\begin{aligned}
& B S_{\alpha}(\vec{r}, t)=\sum_{\vec{x}}\left\langle\bar{q}(\vec{x}+\vec{r}, t) \Gamma q(\vec{x}, t) \mid \Omega_{\alpha}\right\rangle, \\
& \Phi(\vec{r}, t) \quad=B S_{\alpha}(\vec{r}, t) / B S_{\alpha}\left(\overrightarrow{r_{0}}, t\right) .
\end{aligned}
$$

Here the axial-vector channel is calculated with a derivative operator $\Gamma=$ $\sum_{j k}\left(\epsilon_{i j k} \gamma_{j} \partial_{k}\right)$ in order to construct the wave function with large component. The $\left|\Omega_{\alpha}\right\rangle$ is $\alpha$-th energy state which can be extracted by using the eigen functions, $X$, in 

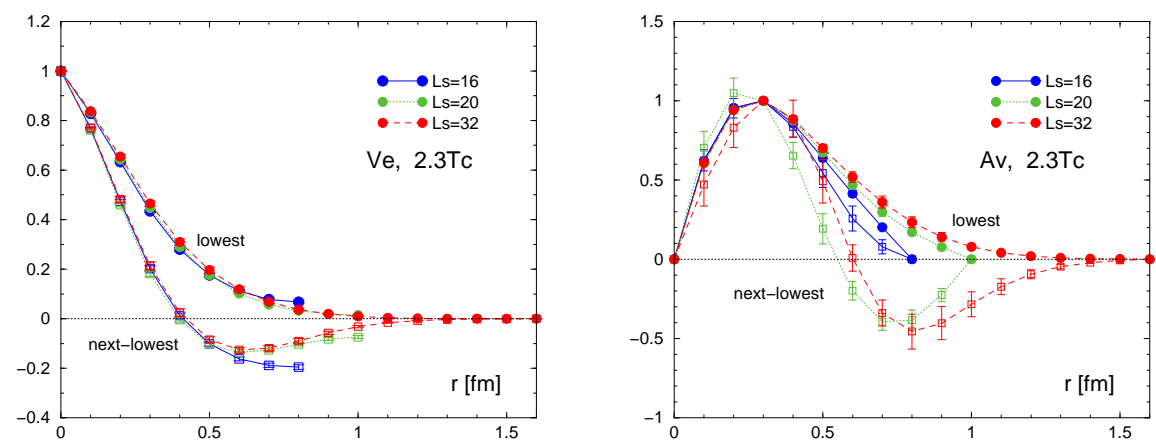

Figure 2. Volume dependences of the lowest and next-lowest state BS wave functions at $T=2.3 T_{c}$. Left and right panels show charmonium with vector and axial-vector channels respectively.

Eq. (1),

$$
\left|\Omega_{\alpha}\right\rangle=O_{\beta}^{\dagger}(\overrightarrow{0}, 0) \mid 0>X_{\beta \alpha} .
$$

We find that the wave functions are stable under the increase of the lattice volume and show clear characteristics of bound state for both channels, even for $2 \mathrm{~S}$ and $2 \mathrm{P}$ excited states.

\section{Conclusion}

In order to study the charmonium dissociation at $T>0$, we extract ground and the first excited charmonium states in S- and P-waves and study boundary condition dependence of their energy, as well as the volume-dependence of their wave functions. From a simulation of quenched anisotropic lattice QCD, we found so far no sign of scattering states up to about $2.3 T_{c}$.

\section{Acknowledgments}

The simulations have been performed on supercomputers (NEC SX-5) at the Research Center for Nuclear Physics (RCNP) at Osaka University. This work is also supported by the Large Scale Simulation Program No.07-18(FY2008) of High Energy Accelerator Research Organization (KEK) and the Nos.17340066 and Nos.19549001 with Grantsin-Aid of the Japanese MEXT.

[1] T. Umeda, R. Katayama, O. Miyamura and H. Matsufuru, Int. J. Mod. Phys. A 16 (2001) 2215 hep-lat/0011085.

[2] T. Umeda, K. Nomura and H. Matsufuru, Eur. Phys. J. C 39S1 (2005) 9 hep-lat/0211003.

[3] S. Datta, F. Karsch, P. Petreczky and I. Wetzorke, Phys. Rev. D 69 (2004) 094507 hep-lat/0312037.

[4] M. Asakawa and T. Hatsuda, Phys. Rev. Lett. 92 (2004) 012001 hep-lat/0308034.

[5] G. Aarts, C. Allton, M. B. Oktay, M. Peardon and J. I. Skullerud, Phys. Rev. D 76, 094513 (2007) arXiv:0705.2198 [hep-lat]].

[6] A. Jakovac, P. Petreczky, K. Petrov and A. Velytsky, hep-lat/0611017

[7] S. Digal, P. Petreczky and H. Satz, arXiv:hep-ph/0110406

[8] F. Karsch, D. Kharzeev and H. Satz, Phys. Lett. B 637, 75 (2006) arXiv:hep-ph/0512239.

[9] T. Umeda, Phys. Rev. D 75 (2007) 094502 hep-lat/0701005.

[10] M. Luscher and U. Wolff, Nucl. Phys. B 339, 222 (1990).

[11] H. Iida, T. Doi, N. Ishii, H. Suganuma and K. Tsumura, Phys. Rev. D 74, 074502 (2006) arXiv:hep-lat/0602008.

[12] H. Matsufuru, T. Onogi and T. Umeda, Phys. Rev. D 64 (2001) 114503 hep-lat/0107001. 